\title{
Motion Control Strategies for Improved Multi Robot Perception
}

\author{
R. Aragues J. Cortes C. Sagues
}

\begin{abstract}
This paper describes a strategy to select optimal motions of multi robot systems equipped with cameras in such a way that they can successively improve the observation of the environment. We present a solution designed for omnidirectional cameras, although the results can be extended to conventional cameras. The key idea is the selection of a finite set of candidate next positions for every robot within their local landmark-based stochastic maps. In this way, the cost function measuring the perception improvement when a robot moves to a new position can be easily evaluated on the finite set of candidate positions. Then, the robots in the team can coordinate based on these small pieces of information. The proposed strategy is designed to be integrated with a map merging algorithm where robots fuse their maps to get a more precise knowledge of the environment. The interest of the proposed strategy for uncertainty reduction is that it is suitable for visual sensing, allows an efficient information exchange, presents a low computational cost and makes the robot coordination easier.
\end{abstract}

\section{INTRODUCTION}

The interest for having groups of robots working cooperatively has rapidly increased.Many tasks cannot be carried out by robots working alone. For other tasks, multi-robot systems offer increased robustness and adaptation. One important task that can be better achieved using a robotic team is the perception of the environment. In these scenarios, it is of interest to optimize individual robot motions to maximize the information collected about the scene. This is the problem that we address in this paper. Robots equipped with vision sensors combine their information and build a local landmark-based stochastic map of the environment. The robots have communication capabilities to exchange their local maps and build a global representation of the environment [1]. However, the construction of this global map may require long communication and computation times. Therefore, it is of interest that robots make decisions based on their local data and small pieces of information received from the other nodes. We focus on strategies that improve the local maps taking into account the proposed improvements of other robots, giving rise to an improvement of the global map. This problem is close to other works on optimization of facilities, see [2], [3], [4], and coverage problems [5], [6], where the objective is to optimally place a group of robots in an environment of interest to achieve maximum coverage. Specifically, it is highly related to exploration guided by information and active sensing.

R. Aragues and C. Sagues are with DIIS - I3A, University of Zaragoza, María de Luna, 50018 Zaragoza, Spain raragues@unizar.es, csagues@unizar.es

J. Cortes is with the Department of Mechanical and Aerospace Engineering, University of California San Diego, 9500 Gilman Dr, La Jolla, California, 92093-0411, USA cortes@ucsd.edu
Many of the existing solutions for exploration and active sensing are based on occupancy grid maps. Here, frontier cells dividing between already explored and unknown sections can be easily detected. Then, robots can evaluate a cost function on this small subset of destinations and make decisions propagating small pieces of information with the other robots. Some examples of these approach are [7], [8], [9] for the single robot case and [10] for multi robot systems. However, the exploration problem turns out to be more complicated for landmark-based maps, since the number of candidate destinations is infinite.

An alternative to the use of sets of candidate vantage locations are global optimization methods [11], [12], [13], where robots search for the best position to reduce the whole map uncertainty. Every robot makes decisions based on its current local estimate of the global map and propagates its observations to the other nodes so that they can update their maps. These approaches result in weak robot coordination, because without a common global map estimate, different robots may end up exploring exactly the same regions. In addition, many of these solutions use gradient methods to find minima on the cost function. Gradient algorithms are computationally expensive since the gradient must be reevaluated at every step. Besides, they may find local minima, and the step size adjustment is complicated.

Exploration and active sensing solutions may also be divided between one step decisions and path planning methods. Most of the previously mentioned works are one step approaches, where robots compute the reduction of the cost function considering exclusively the next robot motion. Approaches based on path planning or trajectory optimization [14], [15] use a larger time horizon and consider the cost function for multiple successive robot motions. Although these strategies may be suitable for a single robot, they present important scaling problems for the multi robot case.

In this paper, we define a method for landmark-based maps which is close to a frontier exploration in the sense that we are able to select a finite set of destinations for every robot. Each robot associates to these positions cost values that can be sent to other robots in the team with the aim of negotiating their next motions. This solution presents multiple appealing features due to its low computation complexity and to the fact that the robots do not need to wait for having a good global map estimate. Instead, they can negotiate on small pieces of information, ensuring that the resulting global map will be improved.

This paper is organized as follows. Section II explains how the robots can compute a prediction of the expected map resulting of placing the robot into vantage locations to sense 
the scene. Section III explains how robots compute these vantage locations for observing features in the map. Then, Section IV presents the strategy for improved perception, explaining both the global and individual cost function to be minimized. Finally, Section V shows the performance of the strategy via simulation.

\section{EXPECTED MAPS FOR THE VANTAGE LOCATIONS}

When a robot moves to a vantage location and takes new measurements of the environment, its local map estimate may become more precise. We compute this expected map applying the same algorithm used by the robots to build their local maps. It is a Simultaneous Localization and Map Building (SLAM) algorithm for bearing only data based on an Extended Kalman Filter (EKF). We use the same measurement and odometry models, and we assume that: a) the measurements are exactly the expected bearings to the features from the new robot pose, and b) the odometry estimate is exactly the new robot pose.

\section{A. Relative Motion Computation}

Let $\hat{\mathbf{x}}_{r}(k)=\left(\hat{x}_{k}, \hat{y}_{k}, \hat{\theta}_{k}\right)^{T}$ be the robot pose at time $k$, $\hat{\mathbf{x}}_{i}(k)=\left(\hat{x}_{i}, \hat{y}_{i}\right)^{T}$ for $i \in\{1, \ldots, m\}$ the feature estimates at time $k$, and $\hat{\mathbf{x}}(k)=\left(\hat{\mathbf{x}}_{r}(k)^{T}, \hat{\mathbf{x}}_{1}(k)^{T} \ldots, \hat{\mathbf{x}}_{m}(k)^{T}\right)^{T}$ the local map estimate at time $k$, with associated covariance $\mathbf{P}(k)$. Let $\mathbf{x}_{g}=\left(x_{g}, y_{g}, \theta_{g}\right)^{T}$ be the goal vantage location where the robot plans to move to. The relative translation and rotation $\mathbf{x}_{k+1}^{k}$ between $\hat{\mathbf{x}}_{r}(k)$ and $\mathbf{x}_{g}$ can be computed as:

$$
\mathbf{x}_{k+1}^{k}=\left(\ominus \hat{\mathbf{x}}_{r}(k)\right) \oplus \mathbf{x}_{g}
$$

where the operator $\ominus$ is the inverse location vector

$$
\ominus \hat{\mathbf{x}}_{r}(k)=\left[\begin{array}{c}
-\hat{x}_{k} \cos \hat{\theta}_{k}-\hat{y}_{k} \sin \hat{\theta}_{k} \\
\hat{x}_{k} \sin \hat{\theta}_{k}-\hat{y}_{k} \cos \hat{\theta}_{k} \\
-\hat{\theta}_{k}
\end{array}\right] \text {. }
$$

The operator $\oplus$ is the composition of two location vectors. It returns a location vector that transforms coordinates between the reference frames $\hat{\mathbf{x}}_{r}(k)$ and $\mathbf{x}_{g}$. Then, the expression for the relative transformation $\mathbf{x}_{k+1}^{k}$ between the robot pose at time $k$ and the goal pose at $k+1$ is:

$$
\mathbf{x}_{k+1}^{k}=\left[\begin{array}{c}
\left(x_{g}-\hat{x}_{k}\right) \cos \hat{\theta}_{k}+\left(y_{g}-\hat{y}_{k}\right) \sin \hat{\theta}_{k} \\
-\left(x_{g}-\hat{x}_{k}\right) \sin \hat{\theta}_{k}+\left(y_{g}-\hat{y}_{k}\right) \cos \hat{\theta}_{k} \\
\theta_{g}-\hat{\theta}_{k}
\end{array}\right] \text {. }
$$

\section{B. EKF Prediction}

The prediction step of the localization and mapping algorithm gives $\overline{\mathbf{x}}(k+1)=\mathbf{x}(k+1 \mid k)=\left(\overline{\mathbf{x}}_{r}(k+1)^{T}, \overline{\mathbf{x}}_{1}(k+\right.$ $\left.1)^{T} \ldots \overline{\mathbf{x}}_{m}(k+1)^{T}\right)^{T}$ and covariance $\mathbf{P}(k+1 \mid k)$ based on the previous state $\hat{\mathbf{x}}(k)$ and covariance $\mathbf{P}(k)$, and the odometry measurements $\mathbf{x}_{k+1}^{k}=\left(x_{\text {odom }}, y_{\text {odom }}, \theta_{\text {odom }}\right)$ with covariance matrix $\mathbf{P}_{\text {odom }}$. Here, $\overline{\mathbf{x}}_{r}(k+1)=\left(\bar{x}_{k+1}, \bar{y}_{k+1}, \bar{\theta}_{k+1}\right)^{T}$ and $\overline{\mathbf{x}}_{i}(k+1)=\left(\bar{x}_{i}, \bar{y}_{i}\right)^{T}$ for $i \in\{1, \ldots, m\}$. The odometry measurements $\mathbf{x}_{k+1}^{k}$ are given by (3), and the odometry noise is modelled as three independent noises in the perpendicular and parallel translations and rotation, $\mathbf{P}_{\text {odom }}=\operatorname{diag}\left(\sigma_{x}^{2}, \sigma_{y}^{2}, \sigma_{\theta}^{2}\right)$, where $\sigma_{x}=K_{x} d$ and $\sigma_{y}=K_{y} d$ are proportional to the translation distance $d=$ $\sqrt{\left(x_{g}-\hat{x}_{k}\right)^{2}+\left(y_{g}-\hat{y}_{k}\right)^{2}}$. The equations used to predict the new state are

$$
\begin{aligned}
\overline{\mathbf{x}}(k+1)= & {\left[\begin{array}{c}
\overline{\mathbf{x}}_{r}(k+1) \\
\bar{x}_{1} \\
\bar{y}_{1} \\
\vdots \\
\bar{x}_{m} \\
\hat{y}_{m}
\end{array}\right]=\left[\begin{array}{c}
\hat{\mathbf{x}}_{r}(k) \oplus \mathbf{x}_{k+1}^{k} \\
\hat{x}_{1} \\
\hat{y}_{1} \\
\vdots \\
\hat{x}_{m} \\
\hat{y}_{m}
\end{array}\right], } \\
& \mathbf{P}(k+1 \mid k)=\mathbf{J}_{1} \mathbf{P}(k) \mathbf{J}_{1}^{T}+\mathbf{J}_{2} \mathbf{P}_{\text {odom }} \mathbf{J}_{2}^{T},
\end{aligned}
$$

where the operator $\oplus$ is the composition of the location vectors $\hat{\mathbf{x}}_{r}(k)$ and $\mathbf{x}_{k+1}^{k}$

$$
\hat{\mathbf{x}}_{r}(k) \oplus \mathbf{x}_{k+1}^{k}=\left[\begin{array}{c}
\hat{x}_{k}+x_{\text {odom }} \cos \hat{\theta}_{k}-y_{\text {odom }} \sin \hat{\theta}_{k} \\
\hat{y}_{k}+x_{\text {odom }} \sin \hat{\theta}_{k}+y_{\text {odom }} \cos \hat{\theta}_{k} \\
\hat{\theta}_{k}+\theta_{\text {odom }}
\end{array}\right],
$$

and $\mathbf{J}_{1}, \mathbf{J}_{2}$ are the Jacobians of the prediction operation relative to, respectively, the map and the odometry measurement:

$$
\begin{aligned}
& \mathbf{J}_{1}=\left[\begin{array}{cc}
\mathbf{j}_{1} & \mathbf{0} \\
\mathbf{0} & \mathbf{I}
\end{array}\right], \quad \mathbf{J}_{2}=\left[\begin{array}{c}
\mathbf{j}_{2} \\
\mathbf{0}
\end{array}\right], \\
& \mathbf{j}_{1}=\left[\begin{array}{ccc}
1 & 0 & -x_{\text {odom }} \sin \hat{\theta}_{k}-y_{\text {odom }} \cos \hat{\theta}_{k} \\
0 & 1 & x_{\text {odom }} \cos \hat{\theta}_{k}-y_{\text {odom }} \sin \hat{\theta}_{k} \\
0 & 0 & 1
\end{array}\right], \\
& \mathbf{j}_{2}=\left[\begin{array}{ccc}
\cos \hat{\theta}_{k} & -\sin \hat{\theta}_{k} & 0 \\
\sin \hat{\theta}_{k} & \cos \hat{\theta}_{k} & 0 \\
0 & 0 & 1
\end{array}\right] .
\end{aligned}
$$

\section{Measurement Prediction}

For every feature with coordinates $\overline{\mathbf{x}}_{i}(k+1)=\left(\bar{x}_{i}, \bar{y}_{i}\right)^{T}$ in the map, the expected bearing measurement with respect to the robot that should be obtained from the predicted robot location $\overline{\mathbf{x}}_{r}(k+1)=\left(\bar{x}_{k+1}, \bar{y}_{k+1}, \bar{\theta}_{k+1}\right)^{T}$ is

$$
\begin{gathered}
h_{i}\left(\overline{\mathbf{x}}_{r}(k+1), \overline{\mathbf{x}}_{i}(k+1)\right)= \\
\operatorname{atan2}\left(\frac{-\left(\overline{\mathrm{x}}_{\mathrm{i}}-\overline{\mathrm{x}}_{\mathrm{k}+1}\right) \sin \bar{\theta}_{\mathrm{k}+1}+\left(\overline{\mathrm{y}}_{\mathrm{i}}-\overline{\mathrm{y}}_{\mathrm{k}+1}\right) \cos \bar{\theta}_{\mathrm{k}+1}}{\left(\overline{\mathrm{x}}_{\mathrm{i}}-\overline{\mathrm{x}}_{\mathrm{k}+1}\right) \cos \bar{\theta}_{\mathrm{k}+1}+\left(\overline{\mathrm{y}}_{\mathrm{i}}-\overline{\mathrm{y}}_{\mathrm{k}+1}\right) \sin \bar{\theta}_{\mathrm{k}+1}}\right) .
\end{gathered}
$$

The Jacobian of the observation model is

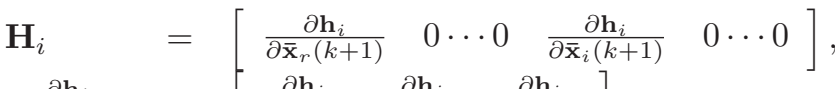

$$
\begin{aligned}
& \frac{\partial \mathbf{h}_{i}}{\partial \overline{\mathbf{x}}_{r}(k+1)}=\left[\begin{array}{lll}
\frac{\partial \mathbf{h}_{i}}{\partial \bar{x}_{k+1}} & \frac{\partial \mathbf{h}_{i}}{\partial \bar{y}_{k+1}} & \frac{\partial \mathbf{h}_{i}}{\partial \bar{\theta}_{k+1}}
\end{array}\right], \\
& \frac{\partial \mathbf{h}_{i}}{\partial \overline{\mathbf{x}}_{i}(k+1)}=\left[\begin{array}{ll}
\frac{\partial \mathbf{h}_{i}}{\partial \bar{x}_{i}} & \frac{\partial \mathbf{h}_{i}}{\partial \bar{y}_{i}}
\end{array}\right],
\end{aligned}
$$

where

$$
\begin{aligned}
& \frac{\partial \mathbf{h}_{i}}{\partial \bar{x}_{k+1}}=-\frac{\bar{y}_{k+1}-\bar{y}_{i}}{\partial \mathbf{h}_{i}}=-\frac{\left.\bar{x}_{k+1}-\bar{x}_{i}\right)^{2}+\left(\bar{y}_{k+1}-\bar{y}_{i}\right)^{2}}{\bar{x}_{k+1}-x_{i}}, \\
& \frac{\partial \mathbf{h}_{i}}{\partial \bar{y}_{k+1}}=\frac{\bar{x}_{k+1}-x_{i}}{\left(\bar{x}_{k+1}-\bar{x}_{i}\right)^{2}+\left(\bar{y}_{k+1}-\bar{y}_{i}\right)^{2}}, \\
& \frac{\partial \mathbf{h}_{i}}{\partial \bar{\theta}_{k+1}}=-1 \text {, } \\
& \frac{\partial \mathbf{h}_{i}}{\partial \bar{x}_{i}}=\frac{\bar{y}_{k+1}-\bar{y}_{i}}{\left(\bar{x}_{k+1}-\bar{x}_{i}\right)^{2}+\left(\bar{y}_{k+1}-\bar{y}_{i}\right)^{2}}, \\
& \frac{\partial \mathbf{h}_{i}}{\partial \bar{y}_{i}}=-\frac{\bar{x}_{k+1}-\bar{x}_{i}}{\left(\bar{x}_{k+1}-\bar{x}_{i}\right)^{2}+\left(\bar{y}_{k+1}-\bar{y}_{i}\right)^{2}},
\end{aligned}
$$


and $\mathbf{h}$ and $\mathbf{H}$ collect the information from all the features in the map

$$
\mathbf{h}=\left[\begin{array}{c}
h_{1} \\
\vdots \\
h_{n}
\end{array}\right], \quad \mathbf{H}=\left[\begin{array}{c}
\mathbf{H}_{1} \\
\vdots \\
\mathbf{H}_{n}
\end{array}\right]
$$

\section{EKF Update}

The final map estimate $\hat{\mathbf{x}}(k+1)=\mathbf{x}(k+1 \mid k+1)$ and covariance $\mathbf{P}(k+1 \mid k+1)$ are obtained as

$$
\begin{array}{ll}
\hat{\mathbf{x}}(k+1) & =\overline{\mathbf{x}}(k+1)+\mathbf{K} \nu, \\
\mathbf{P}(k+1 \mid k+1) & =(I-\mathbf{K H}) \mathbf{P}(k+1 \mid k), \\
\nu & =\mathbf{z}-\mathbf{h}, \\
\mathbf{K} & =\mathbf{P}(k+1 \mid k) \mathbf{H}^{T} \mathbf{S}^{-1}, \\
\mathbf{S} & =\mathbf{H P}(k+1 \mid k) \mathbf{H}^{T}+\mathbf{R},
\end{array}
$$

where $\nu$ is the innovation, $\mathbf{S}$ in the innovation covariance and $\mathbf{K}$ is the Kalman gain. $\mathbf{z}$ is the vector with all the measurements of the features. The matrix $\mathbf{R}$ is the covariance of the observation noise and is equal to $\sigma_{z}^{2} \mathbf{I}$, where $\sigma_{z}$ is the standard deviation of the visual sensor noise.

\section{SELECTION OF VANTAGE LOCATIONS}

In order to manage a finite set of vantage locations for every robot, we adopt a one feature improvement strategy. Every robot in the team will attempt to improve, at least, the observation of one of the landmarks. As a side effect, the observations of other landmarks are also improved. We compute vantage locations where the observation of the landmark produces a high uncertainty reduction. In [16] authors discuss uncertainty minimization within the EKF framework. They show that the minimization of the map uncertainty $\mathbf{P}$ is highly related to the maximization of the covariance of the innovation $\mathbf{S}$. In addition, they show that if the system is driven to the optimal position to obtain maximum information gain, it results in numerically unstable update steps for the EKF. The system becomes more unstable as the robot moves closer to a landmark. To derive the vantage locations to observe the landmarks, we analyze the robot locations that lead to the maximization of $\mathbf{S}$.

For a landmark $i$, the covariance of the innovation $S_{i}$ is (12)

$$
S_{i}=\mathbf{H}_{i} \mathbf{P}(k+1 \mid k) \mathbf{H}_{i}^{T}+\sigma_{z}^{2} .
$$

If we take $\mathbf{P}(k+1 \mid k)=\mathbf{I}$ and apply (9), (10), we can express $S_{i}$ as:

$$
S_{i}=1+\sigma_{z}^{2}+\frac{2}{r^{2}},
$$

where $r=\sqrt{\left(x_{k+1}-x_{i}\right)^{2}+\left(y_{k+1}-y_{i}\right)^{2}}$ is the distance between the robot pose and the landmark. As it can be observed, the maximization of $S_{i}$ is equivalent to the minimization of $r$. Now we introduce into the study the landmark covariance, taking

$$
\mathbf{P}(k+1 \mid k)=\left[\begin{array}{c|cc|c}
\mathbf{I} & \multicolumn{2}{|c|}{\mathbf{0}} & \mathbf{0} \\
\hline \mathbf{0} & P_{x x} & 0 & \mathbf{0} \\
& 0 & P_{y y} & \\
\hline \mathbf{0} & \multicolumn{2}{|c}{\mathbf{0}} & \mathbf{I}
\end{array}\right],
$$

and expressing $P_{y y}=k P_{x x}$, with $k>1$. This models an uncertainty ellipse with its mayor axis perpendicular to the $y$-axis. For robot poses at a constant distance $r, x_{k+1}=$ $r \cos \alpha, y_{k+1}=r \sin \alpha$, the value of $S_{i}$ is:

$$
S_{i}=1+\sigma_{z}^{2}+\frac{1+P_{x x}}{r^{2}}+(k-1) \frac{P_{x x}}{r^{2}} \cos ^{2}(\alpha),
$$

Computing the first and the second derivative of $S_{i}$, the critical points of $S_{i}$ are $\alpha=0+n \pi, n \in \mathbb{Z}$ and $\alpha=$ $\frac{\pi}{2}+n \pi, n \in \mathbb{Z}$. Besides we have that

$$
\frac{\partial^{2} S_{i}}{\partial \alpha^{2}}=2(k-1) \frac{P_{x x}}{r^{2}}\left(1-2 \cos ^{2}(\alpha)\right) .
$$

Since $k>1, S_{i}$ reaches a maximum for $\alpha=0+n \pi, n \in \mathbb{Z}$ and a minimum for $\alpha=\frac{\pi}{2}+n \pi, n \in \mathbb{Z}$. Then we can conclude that $S_{i}$ is maximized when the distance between the robot and the feature is minimized, and that for constant distances, $S_{i}$ is maximized when the landmark is observed from a position in a line perpendicular to its mayor axis.

However, minimizing $r$ may make the system unestable [16]. To avoid this situation, we compute the optimal position for observing a feature so that it lays outside its uncertainty ellipse. It is at a distance from the center so that the angle $\alpha$ between the lines from the vantage point to the extremes of the ellipse must be less than $\frac{\pi}{2}$.

\section{STRATEGY FOR IMPROVED PERCEPTION}

As we mentioned before, our strategy for improved perception is designed to be integrated with a map merging algorithm [1] where robots fuse their local maps into a global map. This process may be executed at every time instant, not necessarily while they are deciding their motions. Given $n$ independent local maps characterized by a mean $\mathbf{x}_{i}$ and a covariance matrix $\mathbf{P}_{i}=\mathbf{P}_{i}(k+1 \mid k+1)$ for $i \in\{1, \ldots, n\}$, the maximum-likelihood estimate $\mathbf{x}_{\text {global }}$ for the global map and its associated covariance matrix $\mathbf{P}_{\text {global }}$ are

$$
\begin{aligned}
\mathbf{x}_{\text {global }} & =\left(\sum_{i=1}^{n} A_{i}^{T} \mathbf{P}_{i}^{-1} A_{i}\right)^{-1} \sum_{i=1}^{n} A_{i}^{T} \mathbf{P}_{i}^{-1} \mathbf{x}_{i}, \\
\mathbf{P}_{\text {global }} & =\left(\sum_{i=1}^{n} A_{i}^{T} \mathbf{P}_{i}^{-1} A_{i}\right)^{-1},
\end{aligned}
$$

where $A_{i}$ are some observations matrices to allow the robots to observe only a portion of the total amount of features. From this expression, we can see that feature estimates with smaller covariances greatly influence their estimates in the global map. Therefore, a precise estimate of a feature can be obtained if, at least, one robot has observed it with high precision.

\section{A. Aggregate Objective Function}

The global cost function $F$ measures the best contributions for the estimate of every feature

$$
\left.F\left(\mathbf{x}_{1}, \cdots, \mathbf{x}_{n}\right)=\sum_{j=1}^{m} \min _{i} f_{i j}\left(\mathbf{x}_{i}\right)\right),
$$


TABLE I

PREDICTED COSTS FOR THE CANDIDATE NEXT MOTIONS FOR ROBOT $i$

\begin{tabular}{|c|ccc|}
\hline & feat 1 & $\cdots$ & feat $m$ \\
\hline $\mathbf{x}_{i}^{\perp}$ & $f_{i 1}\left(\mathbf{x}_{i}^{\perp}\right)$ & $\cdots$ & $f_{i m}\left(\mathbf{x}_{i}^{\perp}\right)$ \\
$\vdots$ & $\vdots$ & & $\vdots$ \\
$\mathbf{x}_{i}^{K}$ & $f_{i 1}\left(\mathbf{x}_{i}^{K}\right)$ & $\cdots$ & $f_{i m}\left(\mathbf{x}_{i}^{K}\right)$ \\
\hline
\end{tabular}

TABLE II

GLOBAL COST FOR A SPECIFIC SELECTION OF NEXT ROBOT POSES

\begin{tabular}{|c|ccc|}
\hline & feat 1 & $\cdots$ & feat $m$ \\
\hline $\mathbf{x}_{1}^{l_{1}}$ & $f_{11}\left(\mathbf{x}_{1}^{l_{1}}\right)$ & $\cdots$ & $f_{1 m}\left(\mathbf{x}_{1}^{l_{1}}\right)$ \\
$\vdots$ & $\vdots$ & & $\vdots$ \\
$\mathbf{x}_{n}^{l_{n}}$ & $f_{n 1}\left(\mathbf{x}_{n}^{l_{n}}\right)$ & $\cdots$ & $f_{n m}\left(\mathbf{x}_{n}^{l_{n}}\right)$ \\
\hline $\min$ & $\min _{i} f_{i 1}\left(\mathbf{x}_{i}^{l_{i}}\right)$ & $\cdots$ & $\min _{i} f_{i m}\left(\mathbf{x}_{i}^{l_{i}}\right)$ \\
\hline
\end{tabular}

where $\mathbf{x}_{i}$ is the next position of the robot $i$ at time $k+1$, for $i \in\{1, \ldots, n\}$ and $f_{i j}$ is the individual cost for the feature $j$ in the local map of the robot $i$ when the next position of the robot $i$ is $\mathbf{x}_{i}$.

The individual cost functions $f_{i j}\left(\mathbf{x}_{i}\right)$ evalute the covariance matrix $\mathbf{P}_{i}\left(\mathbf{x}_{i}\right)$ of the expected map of robot $i$ when it moves to the location $\mathbf{x}_{i}$ (Section II). They measure the uncertainty of the sub-matrix $\left[\mathbf{P}_{i}\left(\mathbf{x}_{i}\right)\right]_{j j}$ within $\mathbf{P}_{i}\left(\mathbf{x}_{i}\right)$ associated to the feature $j$. There exist many metrics for measuring the uncertainty in a covariance matrix. [17] compares metrics based on the determinant, eigenvalues and trace, concluding that all of them perform properly. Here, we select the trace.The individual cost $f_{i j}$ is

$$
\left.f_{i j}\left(\mathbf{x}_{i}\right)\right)=\operatorname{Tr}\left(\left[\mathbf{P}_{i}\left(\mathbf{x}_{i}\right)\right]_{j j}\right) .
$$

\section{B. Coordination Strategy}

The proposed strategy for motion coordination consists of an iterative algorithm where, at every time step, the team of robot performs the following actions:

Optimal position for feature observation: Every robot $i$ computes the best position for observing the $K$ features with higher covariances in its local map, where $K \leq m_{i}$ for $i \in\{1, \ldots, n\}$ is adjusted depending on the performance requirements. As a result, robot $i \in\{1, \ldots, n\}$, obtains $K$ next position candidates which we express as $\mathbf{x}_{i}^{1}, \cdots, \mathbf{x}_{i}^{K}$.

Map prediction: For all the candidate next positions $\mathbf{x}_{i}^{1}, \cdots, \mathbf{x}_{i}^{K}$, robot $i$ computes the predicted map and evaluates the local cost function $f_{i j}\left(\mathbf{x}_{i}^{l}\right)$ for all the features, $l \in\{1, \ldots, K\}, j \in\{1, \ldots, m\}, i \in\{1, \ldots, n\}$. If an estimate of the feature $j$ cannot be found in the local map of robot $i$, then we set $f_{i j}\left(\mathbf{x}_{i}^{l}\right)=\infty$ for all $l \in\{1, \ldots, K\}$. Every robot $i$ can construct a table with the values of $f_{i j}$ (see Table I).

Minimization of the global cost: Given a selected combination of next robot poses $\mathbf{x}_{1}^{l_{1}}, \cdots, \mathbf{x}_{n}^{l_{n}}$, its associated global cost is computed as $\left.\sum_{j=1}^{m} \min _{i} f_{i j}\left(\mathbf{x}_{i}^{l_{i}}\right)\right)$. This is equivalent to sum the values in the last row $(\mathrm{min}$ ) in Table II. The best robot-vantage location assignment is the one minimizing the global cost $F$ (see equation
(19)). Every robot can compute this value, based on the information received from the other robots, and on its own data. This is a classical task-assignment problem where there are $K^{n}$ possible combinations, and one of them produces the best cost. There exist multiple efficient suboptimal methods to solve this problem. Here we just use a brute-force approachs, but in the future we plan to implement a distributed auction-based solution to this problem.

Motion and observation Once the best motions have been decided for the team, the robots move to the new positions, they observe the environment and update their local maps.

The reader may notice that we have not specified when the map merging stage takes place. This is due to the fact that, as we mentioned before, it can be executed in parallel, or even after the robots have finished the exploration.

\section{RESULTS}

In order to show the performance of the algorithm, a simulation has been carried out where a team composed by three robots explore an obstacle-free environment. They estimate their motions based on odometry information and sense the environment using an omnidirectional camera that provides bearing to the landmarks. In the experiments we use an observation noise $\sigma_{z}=1$ degree and an odometry noise $\sigma_{x}=0.01 d, \sigma_{y}=0.01 d, \sigma_{\theta}=2.5$ degrees, where the translation noise is proportional to the travelled distance $d$. In this simulation, the robots process the odometry data and the measurements to construct their local maps using a SLAM algorithm for bearing-only data, see [18] for a detailed description. We initialize every local map with two robot poses to recover the position of some of the landmarks in the map. Their initial maps can be seen in Fig. 1(a). We display in black the ground-truth information, using points for the landmark positions, lines for the robot motions, and triangles for the robot poses. The maps and trajectories estimated by the robots are shown in different colors.

In Fig. 1(a) we show the initial local maps of the robots. Since they have been constructed using two close robot poses, their landmark estimates present high uncertainties. Its associated global map can be found at Fig. 1(b).

At every step, the robots compute their candidate next positions, and evaluate the cost function at these vantage points. In the experiments, we simulate a perfect robot coordination and we provide each robot with its best robotcandidate destination assignment. In Fig. 1(a,c,e) we display these selected motions and the resulting local maps for successive steps. In Fig. 1(b,d,f) we show their associated global map computed by (18).

In this example, we can see the behavior we explained along this paper. If a feature has been observed by multiple robots, and at least one of them has estimated it with high precision, its estimate in the global map presents a small uncertainty. See for instance $F 16$ which has been observed by the three robots; Robot 1 (blue) possess a very uncertain estimate, Robot 3 (red) has a better estimate, although still 
uncertain, and the estimate at Robot 2 (green) is very precise. After merging their maps, the final estimate for $F 16$ is very precise. Therefore, the robots should move to reduce the uncertainties of features which have not been precisely estimated by none of the robots, instead of reducing their local uncertainties.

In Fig. 1(c) we can observe the decisions made by the proposed algorithm and their effects on the global map Fig. 1(d). The robots in the team move to positions that optimize the global knowledge, and as a result, the global map presents a high improvement. They do not exclusively try to reduce their local uncertainties, but instead take care of features uncovered by the team members. See for instance Robot 1 (blue). Its worse feature estimates in its previous local map (Fig. 1(a)), blue, are $F 6$ and $F 16$. He will consider moving to positions where both of them present a reduction on their uncertainties. However, exchanging the information with the other robots, it realizes that one of the team members already possess a precise estimate for $F 16$, and therefore it moves to a extremely bad conditioned pose for the observation of $F 16$, but well conditioned to observe other features. Besides, we can see that the robots tend to move to different regions in the environment, improving the coverage of all the features. In Fig. 1(e-f) we show the next step of the algorithm, where again the robots move to improve the scene perception. Since this global map has reached a high precision, next iterations of the algorithm add no significant improvements.

\section{CONCLUSIONS}

Along this paper we have proposed a motion control strategy for improved perception of a scene capable of efficiently managing landmark-based maps. This strategy is designed to be integrated in a multi robot system, where robots use a map merging algorithm to fuse their maps and get a more precise knowledge of the environment. The described strategy selects a finite set of candidate motions to the robots, and computes its associated cost in the form of the individual contributions of every feature. Therefore, this cost presents a space complexity linear on the map size. This information can be used by the team members to negotiate their next motions, presenting the benefit that robots do not need to wait for having a good global map estimate when they coordinate. In the experiments, we have seen that this approach offers good results in terms of the reduction of both the uncertainties in the local and global maps. Future extensions of this work are in the line of designing distributed coordination strategies for the robots, so that they can negotiate on the information provided by the described algorithm. Other interesting extensions are in the line of considering restricted robot motions, and environments with obstacles.

\section{ACKNOWLEDGMENTS}

This work was supported by projects MEC DPI200607928 and IST-1-045062-URUS-STP

\section{REFERENCES}

[1] R. Aragues, J. Cortes, and C. Sagues, "Distributed map merging in a robotic network," in Proc. of Workshop on Network Robot Systems: human concepts of space and activity, integration and applications, IEEE/RSJ 2008 International Conference on Intelligent RObots and Systems, Nice, France, September 2008, accepted.

[2] A. Okabe, B. Boots, and K. Sugihara, "Nearest neighbourhood operations with generalized Voronoi diagrams: A review," International Journal of Geographical Information Systems, vol. 8, no. 1, pp. 43-71, 1994.

[3] A. Suzuki and Z. Drezner, "The $p$-center location problem in an area," Location Science, vol. 4, no. 1/2, pp. 69-82, 1996.

[4] A. Okabe and A. Suzuki, "Locational optimization problems solved through Voronoi diagrams," European Journal of Operational Research, vol. 98, no. 3, pp. 445-56, 1997.

[5] S. Martínez, F. Bullo, J. Cortés, and E. Frazzoli, "On synchronous robotic networks - Part II: Time complexity of rendezvous and deployment algorithms," in IEEE Conf. on Decision and Control, Seville, Spain, Dec. 2005, pp. 8313-8318.

[6] J. Cortés, S. Martínez, T. Karatas, and F. Bullo, "Coverage control for mobile sensing networks," in IEEE Int. Conf. on Robotics and Automation, Washington, USA, 2002, pp. 1327-1332.

[7] C. Stachniss and W. Burgard, "Mapping and exploration with mobile robots using coverage maps," in Proc. of the IEEE/RSJ International Conference on Intelligent Robots and Systems (IROS), 2003.

[8] T. Tao, Y. Huang, F. Sun, and T. Wang, "Motion planning for slam based on frontier exploration," Mechatronics and Automation, 2007. ICMA 2007. International Conference on, pp. 2120-2125, Aug. 2007.

[9] B. Xi, R. Guo, F. Sun, and Y. Huang, "Simulation research for active simultaneous localization and mapping based on extended kalman filter," Automation and Logistics, 2008. ICAL 2008. IEEE International Conference on, pp. 2443-2448, Sept. 2008.

[10] W. Burgard, M. Moors, C. Stachniss, and F. Schneider, "Coordinated multi-robot exploration," IEEE Transactions on Robotics, vol. 21, pp. 376-386, 2005.

[11] P. Yang, R. Freeman, and K. Lynch, "Distributed cooperative active sensing using consensus filters," Robotics and Automation, 2007 IEEE International Conference on, pp. 405-410, April 2007.

[12] R. Rocha, J. Dias, and A. Carvalho, "Cooperative multi-robot systems a study of vision-based 3-d mapping using information theory," in Proceedings of the 2005 IEEE International Conference on Robotics and Automation, ICRA 2005, Barcelona, Spain, April 2005.

[13] — , "Cooperative multi-robot systems: : A study of vision-based 3-d mapping using information theory," Robotics and Autonomous Systems, vol. 53, no. 3-4, pp. 282-311, 2005.

[14] C. Leung, S. Huang, and G. Dissanayake, "Active slam using model predictive control and attractor based exploration," Intelligent Robots and Systems, 2006 IEEE/RSJ International Conference on, pp. 50265031, Oct. 2006.

[15] _ - "Active slam in structured environments," Robotics and Automation, 2008. ICRA 2008. IEEE International Conference on, pp. 18981903, May 2008.

[16] R. Sim, "Stable exploration for bearings-only slam," in Proceedings of the 2005 IEEE International Conference on Robotics and Automation, ICRA 2005, April 18-22, 2005, Barcelona, Spain, 2005, pp. 24112416.

[17] S. Wenhardt, B. Deutsch, E. Angelopoulou, and H. Niemann, "Active visual object reconstruction using d-, e-, and t-optimal next best views," in 2007 IEEE Computer Society Conference on Computer Vision and Pattern Recognition (CVPR 2007), 18-23 June 2007, Minneapolis, Minnesota, USA, 2007.

[18] R. Aragues and C. Sagues, "Parameterization and initialization of bearing-only information: a discussion," in Proceedings of the Fifth International Conference on Informatics in Control, Automation and Robotics, ICINCO 2008, vol. RA-1, Funchal, Madeira, Portugal, May 2008, pp. 252-261. 


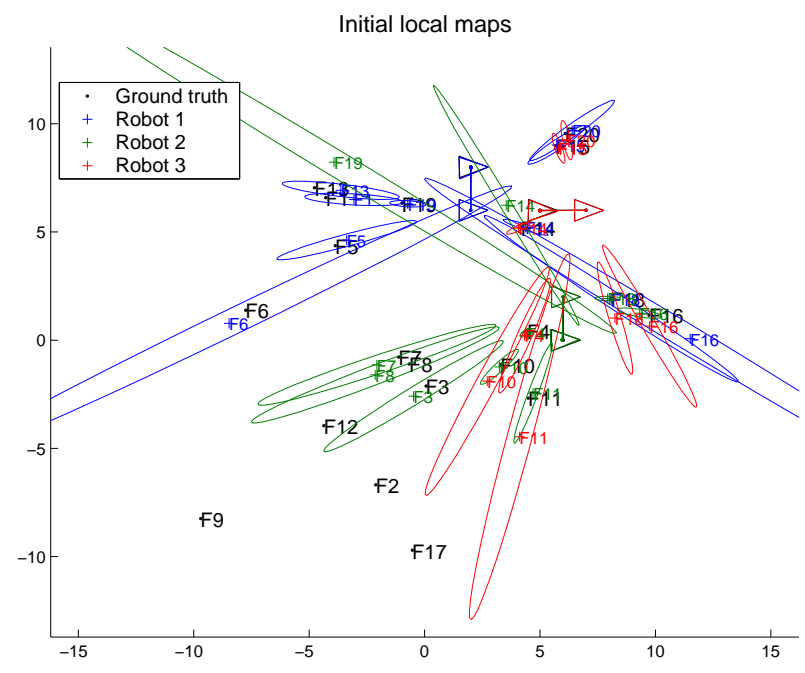

(a)

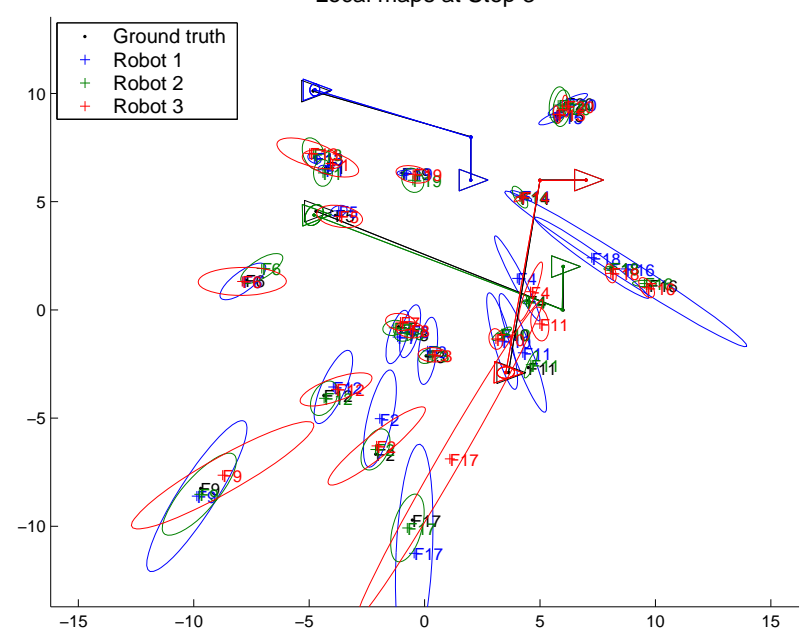

(c)

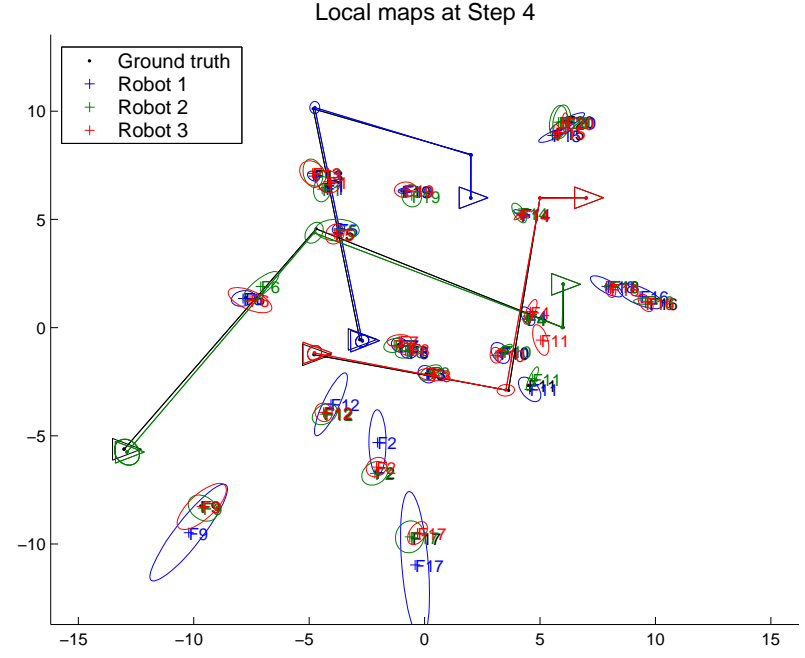

(e)

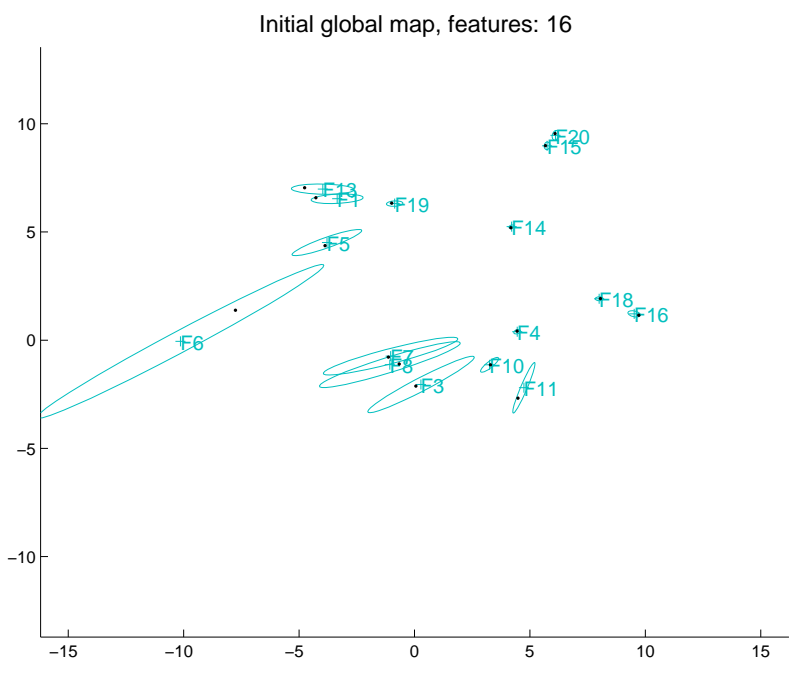

(b)

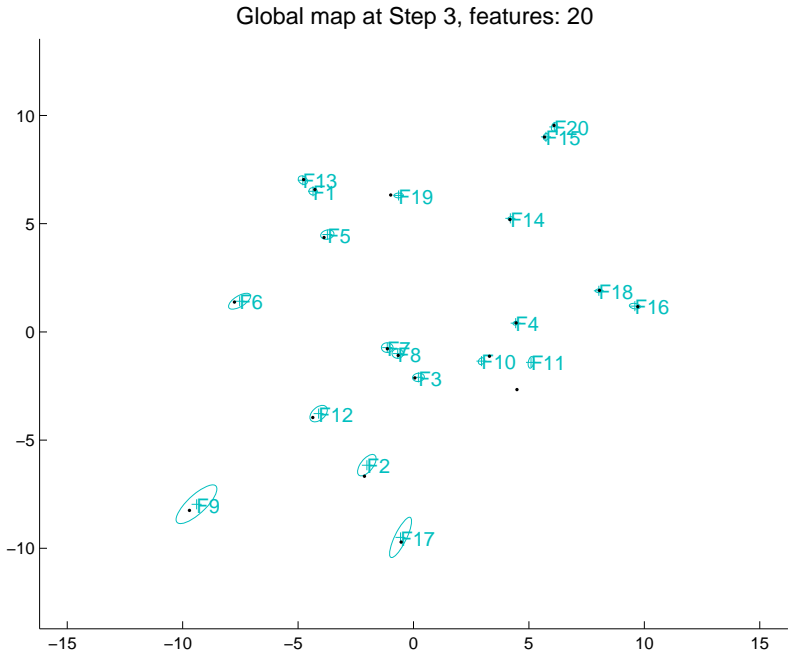

(d)

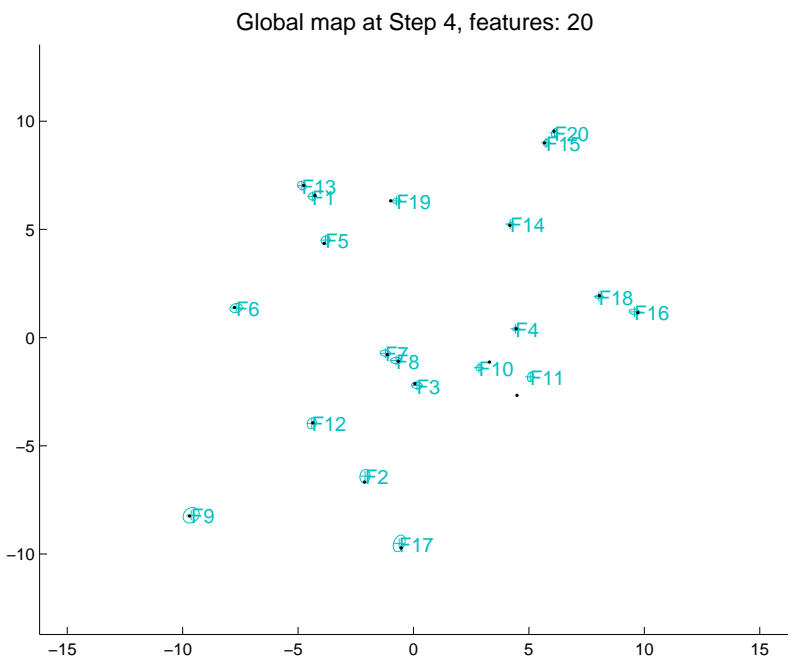

(f)

Fig. 1. Strategy for improved perception. We display some steps of the strategy for improved perception. Black dots are the ground-truth landmark positions. Local map estimates from different robots are shown in a different color. Figures at the right show the maximum-likelihood associated to the local maps. Notice that although the robots do not know this global map, their actions reduce its uncertainty. (a) Initial maps for robots $1,2,3$. (b) Initial global map. (c) Maps for robots 1,2,3 after the first execution of the algorithm. (d) Global map for (c). (e) Maps for robots 1,2,3 after the second execution of the algorithm. (f) Global map for (e). 